\title{
Inventario de Conductas Socialmente Responsables en Universitarios Chilenos
}

\author{
Inventory of Socially Responsible Behaviors in Chilean University Students
}

\author{
Paula Boero $^{1}$, Ítalo Trizano-Hermosilla ${ }^{2}$, Eugenia V. Vinet ${ }^{3}$ y Gracia Navarro ${ }^{4}$
}

\begin{abstract}
Resumen
Se presenta un nuevo instrumento para la medición de la conducta socialmente responsable en universitarios y se da cuenta de sus propiedades psicométricas. Se trabajó con una muestra no probabilística de 1935 estudiantes. Se utilizó un procedimiento de validación cruzada, mediante modelos factoriales -exploratorio y confirmatorio- identificando una estructura latente de cinco factores relacionados, que explican satisfactoriamente los datos obtenidos. Además, se profundizó en las propiedades psicométricas, evaluando modelos de invarianza por sexo y nivel socioeconómico, encontrando niveles de invarianza escalar y diferencias de medias latentes en algunos factores. Se concluye que el inventario presenta evidencia de validez estructural y fiabilidad para su uso en universitarios, permitiendo incluso, comparaciones según sexo y nivel socioeconómico. Se aporta, así, una herramienta psicométricamente adecuada para monitorear y mejorar los niveles de responsabilidad social en estudiantes universitarios. Futuros estudios deberán incorporar variables externas que profundicen en las evidencias de validez de criterio del instrumento.
\end{abstract}

Palabras clave: conducta socialmente responsable, validez, fiabilidad, estructura interna, universitarios

\begin{abstract}
A new instrument for measuring socially responsible behavior in university students is presented, along with its psychometric properties. We worked with a non-probabilistic sample of 1935 students. A cross-validation procedure was used, by calculating exploratory and confirmatory factor models, identifying a latent structure of five related factors that allowed for satisfactorily explaining the data. The psychometric properties of the scale were further assessed, by evaluating models of invariance by sex and socioeconomic levels. Findings show levels of scalar invariance and differences of latent means in some factors. It was concluded that the inventory presents sufficient evidence of psychometric validity and reliability for its use in university students, allowing even for comparisons across sex and socioeconomic levels. Thus, this study provides a tool for future monitoring and improving the levels of social responsibility of university students. Future studies should incorporate external variables that may deepen the evidence of criterion validity for this instrument.
\end{abstract}

Keywords: socially responsible behavior, validity, reliability, internal structure, university students

Este estudio fue financiado por la Comisión Nacional de Investigación Científica y Tecnológica (CONICYT) de Chile, a través del proyecto Fondecyt 1150095. Además, recibió apoyo metodológico del proyecto Fondecyt 11170522.

\footnotetext{
${ }^{1}$ Académica, Departamento de Psicología, Universidad de La Frontera. Av. Francisco Salazar 01145, Temuco, Chile. Tel.: 56-452325621. Correo: paula.boero@ufrontera.cl (correspondencia)

${ }^{2}$ Académico, Departamento de Psicología, Universidad de La Frontera. Av. Francisco Salazar 01145, Temuco, Chile. Tel.: 56-452325612. Correo: italo.trizano@ufrontera.cl

${ }_{3}^{3}$ Académica, Departamento de Psicología, Universidad de La Frontera. Av. Francisco Salazar 01145, Temuco, Chile. Tel.: 56-452325615. Correo: eugenia.vinet@ufrontera.cl

${ }^{4}$ Académica, Departamento de Psicología, Universidad de Concepción. Víctor Lamas 1390. Casilla 160C, Concepción, Chile. Tel.: 56412204996. Correo: gnavarro@udec.cl

Revista Iberoamericana de Diagnóstico y Evaluación - e Avaliação Psicológica. RIDEP · N57 · Vol.4 · 105-118 · 2020

ISSN: 1135-3848 print /2183-6051online
} 


\section{Introducción}

La responsabilidad social (RS) es conceptualizada como la inversión personal en el bienestar de otros y del planeta (Berman, 1990, 1997). En Educación Superior, la RS ha sido promovida, desde 1998, por la Organización de las Naciones Unidas para la Educación, la Ciencia y la Cultura (UNESCO), en la Declaración de la Conferencia Mundial de Educación Superior, afirmando que la RS es un compromiso de todos los actores involucrados en educación incluidos los gobiernos. Específicamente la Declaración de la UNESCO señala que, la Educación Superior no solo debería proveer competencias sólidas al mundo presente y futuro, sino que debe contribuir a la educación de ciudadanos éticos, comprometidos con la construcción de la paz, la defensa de los derechos humanos y los valores de la democracia (UNESCO, 1998). Asimismo, el Proyecto Tuning Latinoamérica ha considerado, desde 2007, a la "responsabilidad social y el compromiso ciudadano" dentro de las 27 competencias genéricas prioritarias para la Educación Superior, vinculando la RS a la misión de crear conocimiento y formar científicos, humanistas y profesionales que se orienten a satisfacer las necesidades de desarrollo de un país (Tuning América Latina, 2007).

Estos imperativos están intrínsecamente relacionados a concepciones filosóficas y psicológicas fundantes del principio de RS. Desde un punto de vista filosófico, la RS se funda en el valor de la responsabilidad, comprendida como "la capacidad y el deber de un sujeto de reconocer y aceptar las consecuencias de sus actos moral, civil o penalmente." (Lalande, 1966, p. 926). Los fundamentos éticos sobre responsabilidad, que dan sustento al principio de RS, se han desarrollado desde Aristóteles en adelante. Según Carbonero, Martín-Antón, Otero y Monsalvo (2017), para Aristóteles, la responsabilidad puede ser pensada como un valor universal con una base cognitiva o como un valor que depende de las condiciones culturales e históricas. Siguiendo esta concepción, la responsabilidad exige, no solo el conocimiento intelectual del bien, sino también una conducta virtuosa. Es decir, que las personas realicen actos virtuosos, en este caso, actos responsables que cumplan dos condiciones: a) que sean voluntarios y b) que sean producto de una libre elección (Bravo, 2006). El filósofo alemán Hans Jonas (2006), amplía el concepto sosteniendo una ética de la responsabilidad orientada hacia el futuro. Jonas muestra la problemática de la responsabilidad humana frente a las consecuencias del desarrollo científico tecnológico y del ilimitado poder que han alcanzado los hombres sobre el mundo natural y social, enfatizando los peligros que implica el desarrollo actual de la técnica para la supervivencia humana. Ante este diagnóstico critica el abandono de la responsabilidad a su dimensión puramente verbal o formal, planteando la imperiosa necesidad de analizar la problemática de la responsabilidad para la vida futura de la humanidad y formula el imperativo ético "actúa de tal modo, que las consecuencias de tu acción sean compatibles con la permanencia de una verdadera vida humana sobre la tierra" (Jonas, 2006, p.47). De esta forma, Jonas sienta las bases de la RS mostrando que el principio de responsabilidad tiene un alcance no sólo individual sino también colectivo y prospectivo. Es decir, es aquella responsabilidad capaz de prever las consecuencias de las acciones que aún no se han emprendido y que, por situarse en el futuro, debe tener una intención hacia el bien común y el bienestar de otros y del planeta.

Complementariamente, desde una perspectiva psicológica individual, Sheldon Berman (1997), señala que la RS es un compromiso personal con los demás que comprende tres dimensiones: 1) Comprender que cada persona pertenece a una red social más amplia; 2) Mantener relaciones interpersonales basadas en consideraciones éticas de justicia y de preocupación por los otros; y 3) Actuar con integridad de manera consistente con los propios valores.

La primera de ellas, inspirada en el modelo ecológico de Bronfenbrenner (1979), se refiere al sentido de conexión e interdependencia con otros. Se produce desde una escala local a una escala global y es decisiva en la construcción de la propia identidad. La segunda dimensión, alude a la presencia de dos voces morales: una sustentada por Piaget (1932/1994) y Kohlberg (1984) y la otra por Gilligan (1982, 1988). Ambas son complementarias, pero de naturaleza diferente. Según Berman (1997) estas dos dimensiones 
llaman la atención sobre la vulnerabilidad de las personas, experimentada tanto por la opresión como por el abandono. La voz de la justicia reivindica las inequidades y, a menudo, se expresa en reglas, principios y obligaciones. La voz del cuidado, en cambio, se orienta hacia la sensibilidad por el sufrimiento y, a menudo, se manifiesta en términos de compasión y respuestas frente a las necesidades de otros. Para Berman (1997), la RS encierra un balance de ambas, que cuida el bienestar de otros y de la comunidad como un todo. Por último, la tercera dimensión, es actuar con integridad, consistentemente con los propios valores, en otras palabras, es la inversión personal en el bienestar propio, de otros y del planeta. Según Kohlberg y Candee (1984), la RS es un atributo que denota una preocupación por otros y la aceptación de las consecuencias de las propias acciones, así como también, consistencia entre lo que se declara, lo que se debería hacer y lo que efectivamente se hace, visualizando que nuestras acciones tienen implicancias políticas y sociales.

En un plano más concreto Navarro (2006, 2012), señala que, como la RS es una abstracción, un valor, es necesario concretarlo a través de una conducta moral, esto es, mediante la realización de actos virtuosos que apunten en dirección al bien, a la supervivencia y a la felicidad del hombre y de la especie humana. Luego, define la conducta socialmente responsable (CSR), como el conjunto de acciones morales concretas por medio de las cuales se ejerce la responsabilidad social (Navarro, 2012). Estas incluyen conductas prosociales, cooperativas (Crocetti, Jahromi, \& Meeus, 2012), de participación (Navarro, 2012), de autoeficacia (Oros, 2017) y de autocontrol (Navarro, 2012). Ellas, se ejercen en diferentes ámbitos y tienen a la base una intención orientada hacia el bienestar común. La conducta pro-social se refiere a las acciones voluntarias que tienen la intención de beneficiar o ayudar a un individuo o grupo de individuos (Eisenberg \& Mussen, 1989). La conducta de cooperación, implica la opción voluntaria de obrar conjuntamente con otro u otros, teniendo como foco el bien común. La conducta participativa, en tanto, consiste en tomar parte en acciones o decisiones que impactan o tienen una influencia en el bien común (Navarro, 2012). La conducta de autoeficacia, es definida como "la evaluación que realizamos sobre nuestra capacidad de realizar con éxito determinadas actividades o tareas" (Oros, p.173, 2017); por último, la conducta de auto-control es comprendida como la capacidad de una persona para mantener sus metas, postergar la gratificación y suprimir aquellos impulsos que resultan perjudiciales (Churchland \& Suhler, 2014). En resumen, la CSR implica alcanzar la capacidad de conciliar la satisfacción de las propias necesidades con la satisfacción de necesidades de otros (Navarro, 2012).

Si para la Educación Superior, la RS es un imperativo, es necesario medir este comportamiento de forma válida y confiable, de manera que sirva de referente para monitorear el desarrollo de esta competencia genérica en población universitaria y emprender estrategias para gestionarla.

A la fecha, el único instrumento disponible para medir esta competencia, en habla hispana y para universitarios, es el Cuestionario de Autoatribución de Comportamientos Socialmente Responsables (CACSR) elaborado por Davidovich, Espina, Navarro y Salazar (2005). El instrumento constaba originalmente de 10 dimensiones, 40 ítems y 2 escalas: una de frecuencia del comportamiento, y otra que mide su intención subyacente. En un estudio piloto, se validó el instrumento mediante el método interjueces y se obtuvieron altos índices de consistencia interna mediante el coeficiente alfa de Cronbach (Davidovich et al., 2005). Posteriormente, Navarro, Pérez y Espina (2012), realizaron un análisis de la estructura factorial del instrumento mediante modelos exploratorios, trabajando con una matriz de correlación $\mathrm{r}$ de Pearson, método de estimación de ejes principales y una rotación oblimin, reduciendo a 30 el número de ítems y obteniendo empíricamente tres dimensiones: a) Participación Social, referida a la inmersión activa en la sociedad mediante actividades informativas, colaborativas y asistenciales $(\alpha=.76)$; b) Participación Religiosa, definida como la inclusión en actividades religiosas y promoción de estas $(\alpha=.89)$; y c) Respeto hacia el Entorno, descrita como la relación con el entorno social y físico caracterizado por buscar su preservación y bienestar $(\alpha=.71)$. Sin embargo, ha resultado 
necesario elaborar un nuevo instrumento a partir de las versiones previas, por varias razones: En primer lugar, debido a la complejidad del constructo de RS, se ha requerido un ajuste progresivo de sus ítems y respectivas dimensiones, ya que se modificó el instrumento original, en varios aspectos: se cambiaron las instrucciones, se cambió la redacción de los ítems y la modalidad de respuesta. Es decir, estamos ante un instrumento nuevo. En segundo lugar, se han producido desarrollos estadísticos recientes que aportan mayor precisión a los análisis del nuevo instrumento. En tercer lugar, han mudado las características evolutivas de los estudiantes y, en cuarto lugar, por la importancia que, actualmente, tiene esta competencia genérica para la formación universitaria. Así, el objetivo general de este estudio es construir un nuevo instrumento de medición de la RS para estudiantes universitarios y dar cuenta de sus propiedades psicométricas. En términos específicos, se evaluará la estructura factorial del nuevo instrumento mediante análisis factorial exploratorio y confirmatorio, y se estimarán los niveles de precisión con los que se mide cada una de las dimensiones involucradas. Complementariamente, se evaluará el grado de invarianza que presenta el instrumento según variables sociodemográficas tales como sexo y nivel socioeconómico (NSE). Para diferenciar este nuevo instrumento de los anteriores, se le denominará Inventario de Conducta de Socialmente Responsable en Universitarios (ICOSORE-U) (Anexo A).

\section{Método}

\section{Participantes}

Mediante un muestreo intencionado se encuestaron 1935 estudiantes universitarios, provenientes de cuatro universidades chilenas del norte, centro y sur del país. Los participantes tenían entre 18 y 29 años de edad, con un promedio de 21.30 ( $\mathrm{DE}=2.04$ ). Un $52.2 \%$ era mujer. Un $58.3 \%$ pertenecía al NSE medio-bajo y el $41.7 \%$ restante al NSE medio-alto. El 68.6\%, vivía con sus padres y un $78.7 \%$ no trabajaba. El $0.5 \%$ reportó estar casado y el $4.6 \%$ señaló tener hijos.

\section{Instrumentos}

Se administró una batería constituida por un cuestionario socio-demográfico y un conjunto de seis instrumentos, orientados a caracterizar a los participantes en variables socioculturales y de salud mental. Entre ellos, se incluyó la versión en estudio del ICOSORE-U. Esta versión mejoró la redacción de algunos ítems de las versiones previas del CACSR y eliminó un ítem que contenía una doble negación que dificultaba su comprensión. Además, modificó las instrucciones de administración desde una sentencia que preguntaba acerca de "la autoatribución de un determinado comportamiento", hacia una instrucción que indagó sobre "la conducta efectivamente ejecutada en el lapso de los últimos 6 meses". Mantuvo el formato tipo Likert de cuatro opciones en donde 0 corresponde a Nunca y 4 a Siempre.

\section{Procedimiento}

Para la administración del instrumento en estudio se contactó a las direcciones académicas de las universidades participantes para programar la toma de datos. A los estudiantes se les dio a conocer los objetivos del estudio y se les invitó a participar de forma voluntaria. Quienes accedieron a participar, firmaron un consentimiento informado, visado por el Comité de Ética institucional y respondieron utilizando un código alfanumérico de identificación para conservar el anonimato.

\section{Análisis de Datos}

En primer lugar, se realizaron análisis descriptivos de los 29 ítems del instrumento en estudio, obteniendo medidas de tendencia central, dispersión y forma. Para evaluar las evidencias de validez se realizó, mediante validación cruzada, un análisis factorial exploratorio con la primera mitad de la muestra para identificar la estructura latente del inventario, para ello se siguió las recomendaciones de Ledesma, Ferrando y Tosi (2019), por lo que se trabajó con la matriz de correlación policórica, el método de estimación de Mínimos Cuadrados no Ponderados (ULS) y una rotación oblimin al considerar que los factores se encuentran relacionados. Una vez identificada la estructura se procedió, con la segunda mitad de la 
muestra, a efectuar un análisis factorial confirmatorio contrastando el modelo identificado previamente. Para ello se trabajó con la matriz de correlaciones policóricas, recomendada para trabajar con ítems tipo Likert, especialmente en caso de que presenten altos niveles, en valor absoluto, de asimetría y/o curtosis, junto a pocas categorías de respuesta (Asun, Rdz-Navarro, \& Alvarado, 2016; Flora, LaBrish, \& Chalmers, 2012) y con el método de estimación de Mínimos Cuadrados Ponderados con media y varianza ajustada (WLSMV). Para evaluar la calidad de cada modelo de reproducir los datos, se consideraron los siguientes índicadores: índice de ajuste comparativo (CFI), índice de Tucker-Lewis (TLI) y error cuadrático medio de aproximación (RMSEA), junto a su intervalo de confianza al 90\%. Para CFI y TLI se consideró un ajuste suficiente del modelo los valores superiores a .90, un ajuste adecuado valor superiores a .95 (Schreiber, Nora, Stage, Barlow, \& King, 2006), mientras que para RMSEA se consideró un ajuste adecuado los valores inferiores a .08 o $\quad .06$ (Schreiber et al., 2006). Estos análisis se realizaron mediante el software Mplus 7.1.

Posteriormente, en base a comparaciones de estudios previos (Navarro et al., 2010) se estimó el grado de invarianza factorial, entre hombres y mujeres; y también, por NSE medio-bajo y medioalto, para el modelo de cinco factores evaluando los niveles de: a) Configuración; b) Métrico; c) Escalar y finalmente, se compararon las medias latentes de los factores. Para comparar estos niveles, se utilizó el comando DIFFTEST y la diferencia en los índices de ajuste CFI $(\triangle \mathrm{CFI})$,

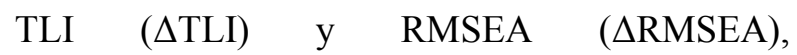
considerándose como evidencia de un adecuado ajuste para el modelo más restrictivo valores menores a .01 para $\Delta$ CFI y $\Delta$ TLI y menores a .015 para $\triangle$ RMSEA (Kueh, Abdullah, Kuan, Morris \& Naing, 2018; Putnick \& Bornstein, 2016).

Considerando las limitaciones del coeficiente alfa para estimar el nivel de fiabilidad de los instrumentos de medida en psicología (Revelle \& Zinbarg, 2009; Sijtsma, 2009; Trizano-Hermosilla \& Alvarado, 2016; Yang \& Green, 2011), se utilizaron, además, los siguientes estimadores: Omega (Reise, 2012; Zinbarg, Revelle, Yovel, \& Li, 2005; Zinbarg, Yovel, Revelle, \& McDonald, 2006) y el Greatest Lower Bound (GLB). Las estimaciones de omega se obtuvieron de las soluciones factoriales, mientras GLB y alfa, junto a su intervalo de confianza al $95 \%$ se estimaron con el software JASP 0.9.2.

\section{Resultados}

\section{Análisis Descriptivos}

En la Tabla 1 se presentan los estadísticos de tendencia central, dispersión y forma de los ítems que conforman el inventario. En general se aprecian altos niveles de asimetría, tanto positiva como negativa y altos niveles de curtosis en los ítems.

\section{Evidencias de Estructura Interna}

\section{Análisis Exploratorios}

Se realizaron Análisis Factoriales Exploratorios con la primera mitad de la muestra (n1=968) identificando una estructura coherente de cinco factores latentes, si bien, el análisis paralelo, sugirió una estructura de tres factores esta fue desestimada debido a que la estructura de cinco factores representaba mejor las relaciones entre el conjunto de ítems y las respectivas dimensiones del nuevo instrumento (ver Tabla 2 y Anexo A). En este proceso, se empleó análisis de contenidos bajo las premisas de la teorización de la RS. Concordante con ello, el ítems 5 "Cuidé de mi salud mediante una alimentación equilibrada, deporte, controles médicos, evitar fumar, entre otros."; el ítem 12 "Escuché con atención a los demás cuando hablaban."; el ítem 13 " Asistí a actividades que complementan mi formación profesional como charlas, seminarios o cursos relacionados con mi carrera"; y el ítem 18 "Leí libros no relacionados con lo académico", no muestran coherencia conceptual ni con el constructo de RS ni tampoco con la estructura de cinco factores identificados. Además, presentaron saturaciones menores al umbral fijado previamente. Por tanto, se decidió su eliminación.

Los cinco factores identificados fueron nombrados como: Participación Religiosa (ítems 4, 11, 16 y 22), Responsabilidad Académica (ítems 2, 9, 15 y 20), Convivencia Social (7, 8, 14, 19, 23, 25, 29), Participación en Ayuda Social (1, 3, 10, 21, 26) y Participación Ciudadana y Cultural (ítem 
Tabla 1. Estadísticos descriptivos de los ítems del ICOSORE-U

\begin{tabular}{|c|c|c|c|c|c|}
\hline Items & Media & Mediana & Desv. típ. & Asimetría & Curtosis \\
\hline 01 & 1.05 & 1.00 & 1.169 & .923 & -.079 \\
\hline 02 & 3.79 & 4.00 & .491 & -2.937 & 12.022 \\
\hline 03 & .56 & .00 & .947 & 1.794 & 2.623 \\
\hline 04 & .61 & .00 & 1.228 & 1.923 & 2.279 \\
\hline 05 & 2.56 & 3.00 & 1.166 & -.557 & -.441 \\
\hline 06 & 2.87 & 3.00 & 1.079 & -.714 & -.203 \\
\hline 07 & 3.69 & 4.00 & .774 & -3.048 & 9.780 \\
\hline 08 & 3.18 & 4.00 & 1.213 & -1.396 & .840 \\
\hline 09 & 3.45 & 4.00 & .673 & -1.095 & 1.269 \\
\hline 10 & .70 & .00 & 1.055 & 1.540 & 1.598 \\
\hline 11 & .59 & .00 & 1.186 & 1.970 & 2.559 \\
\hline 12 & 3.36 & 3.00 & .698 & -1.026 & 1.485 \\
\hline 13 & 1.62 & 2.00 & 1.245 & .305 & -.868 \\
\hline 14 & 2.96 & 3.00 & .958 & -.746 & .185 \\
\hline 15 & 3.21 & 3.00 & .853 & -1.006 & .799 \\
\hline 16 & .29 & .00 & .829 & 3.141 & 9.357 \\
\hline 17 & .70 & .00 & 1.150 & 1.601 & 1.508 \\
\hline 18 & 1.78 & 2.00 & 1.402 & .214 & -1.202 \\
\hline 19 & 3.63 & 4.00 & .777 & -2.580 & 7.098 \\
\hline 20 & 3.22 & 3.00 & .816 & -1.034 & 1.140 \\
\hline 21 & .49 & .00 & .932 & 2.043 & 3.572 \\
\hline 22 & .56 & .00 & 1.074 & 1.884 & 2.446 \\
\hline 23 & 3.60 & 4.00 & .692 & -1.968 & 4.420 \\
\hline 24 & 1.51 & 1.00 & 1.497 & .499 & -1.201 \\
\hline 25 & 3.59 & 4.00 & .784 & -2.380 & 6.227 \\
\hline 26 & 1.05 & 1.00 & 1.206 & .907 & -.222 \\
\hline 27 & 2.80 & 3.00 & 1.084 & -.692 & -.166 \\
\hline 28 & 1.67 & 2.00 & 1.317 & .243 & -1.061 \\
\hline 29 & 1.84 & 2.00 & 1.269 & .128 & -.989 \\
\hline
\end{tabular}

$6,17,24,27$ y 28). Estos factores presentaron niveles de correlación de moderados a bajos entre sí, evidenciando su naturaleza multidimensional (ver Tabla 3).

\section{Análisis Confirmatorios}

Posteriormente, se procedió a evaluar la estructura identificada en el paso anterior mediante un modelo factorial confirmatorio. En la Figura 1 se presentan las saturaciones del modelo evaluado, así como las relaciones entre los factores. El ajuste del modelo de cinco factores fue excelente $\chi^{2} \quad(265)=1089.253, \quad p<.001$, $\mathrm{CFI}=.960$, TLI $=.955$, RMSEA $=.057(.053-.060)$, aceptándose la estructura latente previamente evaluada.
Modelos de invarianza según sexo y según NSE

$\mathrm{Al}$ evaluar el grado de invarianza factorial entre hombres y mujeres se observan adecuados índices de ajuste para cada uno de los modelos de invarianza testeados (ver Tabla 4). Al comparar el modelo de invarianza métrica con el de configuración se puede mantener la hipótesis nula de igualdad entre ambos modelos $(p=.06)$, por su parte, al cotejar el modelo de invarianza escalar con el de invarianza métrica se rechaza la hipótesis nula de igualdad mediante la diferencia de chi-cuadrado $(p<.001)$, sin embargo, si se consideran las mínimas diferencias en los otros índices de ajuste, es posible mantener la equivalencia entre ambos modelos y considerar un nivel de invarianza escalar entre ambos grupos. $\mathrm{Al}$ comparar las medias latentes, dentro del modelo 
Tabla 2. Solución Factorial Exploratoria de cinco factores

\begin{tabular}{|c|c|c|c|c|c|}
\hline Items & $\begin{array}{l}\text { Participación } \\
\text { Religiosa }\end{array}$ & $\begin{array}{l}\text { Responsabilidad } \\
\text { Académica }\end{array}$ & $\begin{array}{l}\text { Convivencia } \\
\text { Social }\end{array}$ & $\begin{array}{c}\text { Participación en Ayuda } \\
\text { Social }\end{array}$ & $\begin{array}{c}\text { Participación } \\
\text { Ciudadana } \\
\text { y Cultural }\end{array}$ \\
\hline 01 & -.011 & $-.351 *$ & $.332 *$ & $.410 *$ & -.005 \\
\hline 02 & .068 & $.644 *$ & .028 & -.065 & .134 \\
\hline 03 & $.148 *$ & -.008 & -.057 & $.555 *$ & $.184 *$ \\
\hline 04 & $.962 *$ & .034 & -.038 & .003 & -.015 \\
\hline 06 & -.031 & $.256^{*}$ & .044 & -.052 & $.465^{*}$ \\
\hline 07 & -.074 & .061 & $.590 *$ & -.009 & -.079 \\
\hline 08 & .045 & $-.214 *$ & $.803 *$ & .045 & -.068 \\
\hline 09 & -.028 & $.711 *$ & .042 & $.286^{*}$ & -.078 \\
\hline 10 & .015 & .015 & -.008 & $.744 *$ & $.228^{*}$ \\
\hline 11 & $.995 *$ & $.064 *$ & -.023 & $-.074 *$ & .027 \\
\hline 14 & .022 & .061 & $.526 *$ & .011 & $.104 *$ \\
\hline 15 & .033 & $.575 *$ & .052 & .082 & $.191 *$ \\
\hline 16 & $.941 *$ & $-.085^{*}$ & .006 & .062 & .016 \\
\hline 17 & .018 & -.042 & -.067 & .043 & $.708 *$ \\
\hline 19 & -.013 & .055 & $.453 *$ & -.038 & .014 \\
\hline 20 & -.019 & $.577 *$ & -.015 & $.243 *$ & -.117 \\
\hline 21 & $.134 *$ & .027 & .035 & $.624 *$ & .015 \\
\hline 22 & $.885 *$ & -.028 & $.101 *$ & .025 & -.052 \\
\hline 23 & -.028 & $.193 *$ & $.445 *$ & -.015 & .096 \\
\hline 24 & -.011 & -.006 & .001 & .092 & $.637 *$ \\
\hline 25 & -.003 & .081 & $.729 *$ & $-.149 *$ & .068 \\
\hline 26 & -.014 & .001 & -.001 & $.633 *$ & $.294 *$ \\
\hline 27 & -.013 & $.147 *$ & .081 & -.039 & $.448 *$ \\
\hline 28 & -.001 & $-.187 *$ & .069 & .119 & $.527 *$ \\
\hline 29 & -.010 & $-.248 *$ & $.458 *$ & .029 & .090 \\
\hline
\end{tabular}

Tabla 3. Correlación entre los factores del ICOSORE-U en el modelo AFE

\begin{tabular}{|c|c|c|c|c|c|}
\hline & $\begin{array}{l}\text { Participación } \\
\text { Religiosa }\end{array}$ & $\begin{array}{l}\text { Responsabilidad } \\
\text { Académica }\end{array}$ & $\begin{array}{l}\text { Convivencia } \\
\text { Social }\end{array}$ & $\begin{array}{c}\text { Participación en Ayuda } \\
\text { Social }\end{array}$ & $\begin{array}{c}\text { Participación } \\
\text { Ciudadana y } \\
\text { Cultural } \\
\end{array}$ \\
\hline $\begin{array}{l}\text { Participación } \\
\text { Religiosa }\end{array}$ & 1 & & & & \\
\hline $\begin{array}{l}\text { Responsabilidad } \\
\text { Académica }\end{array}$ & -.036 & 1 & & & \\
\hline Convivencia Social & -.080 & $.410 *$ & 1 & & \\
\hline $\begin{array}{l}\text { Participación en } \\
\text { Ayuda Social }\end{array}$ & $.358^{*}$ & -.138 & $.119 *$ & 1 & \\
\hline $\begin{array}{l}\text { Participación } \\
\text { Ciudadana y Cultural }\end{array}$ & .091 & -.003 & $.297 *$ & $.355^{*}$ & 1 \\
\hline
\end{tabular}

escalar, fijando las medias de las mujeres a $0 \mathrm{y}$ estimando libremente las medias de los hombres, se observan valores inferiores para los hombres en las dimensiones de Participación Religiosa (-.0231, $p=.021)$, Responsabilidad Académica (-.251, $p<.001)$, Participación en Ayuda Social $(-.109, p<.001)$ y Convivencia
Social (-.0173, $p<.001)$, sin embargo no se encontraron diferencias estadísticamente significativas en Participación Ciudadana y Cultural $(p=.774)$.

Por su parte, al comparar el grado de invarianza entre los NSE medio-bajo y medio-alto (ver Tabla 4), se observan valores satisfactorios en 


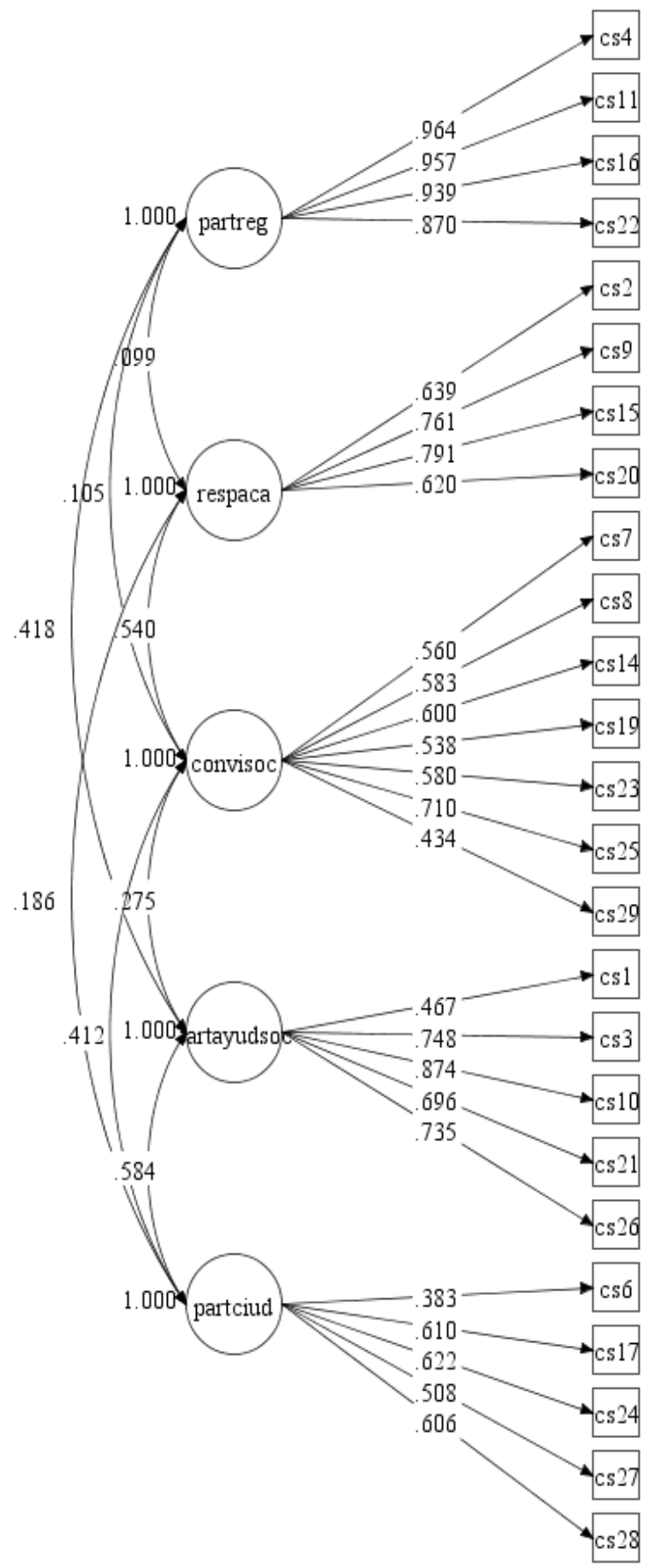

Figura 1. Modelo factorial confirmatorio de cinco dimensiones del ICOSORE-U en estudio

los índices de ajuste para cada uno de los modelos. Al comparar el modelo métrico con el de configuración se mantiene la hipótesis nula de equivalencia entre los dos $(p=.321)$, en tanto al comparar entre los modelos métrico y escalar se observa un valor $p$ muy cercano al puntaje de corte (.044) y considerando las mínimas diferencias en los índices de ajuste, se puede argumentar la equivalencia entre ambos niveles socioeconómicos hasta el nivel de invarianza escalar. Al realizar las comparaciones de las medias latentes, fijando en 0 las medias para el nivel socioeconómico medio-bajo se observa menor Participación Religiosa en el nivel medio-alto $(-.309, p=.004)$, mayor Convivencia Social $(.106, p=.009)$ y mayor Participación Ciudadana $(.089, p=.001)$.

\section{Fiabilidad}

En la Tabla 5 se muestran las estimaciones de la fiabilidad para cada una de las dimensiones del inventario, evaluadas mediante los coeficientes: omega de McDonald, GLB y alfa de Cronbach junto a su intervalo de confianza al 95\%. La dimensión de Participación Religiosa presenta el nivel de fiabilidad más elevado, superior a .90 con todos los coeficientes; las otras dimensiones presentan niveles moderados de fiabilidad, siendo la más baja la dimensión de Participación Ciudadana y Cultural, con valores levemente superiores a .60.

\section{Discusión}

El objetivo de este estudio fue construir un nuevo inventario de medición de la RS para estudiantes universitarios, inspirado en las versiones de referencia (Davidovich, et al., 2005; Navarro et al., 2012) y dar cuenta de sus propiedades psicométricas. Para diferenciarlo de las versiones previas a este nuevo instrumento de medida se lo denominó ICOSORE-U o Inventario de Conductas Socialmente Responsables en Universitarios. Específicamente se evaluó la estructura factorial y se estimaron los niveles de precisión de cada una de las dimensiones involucradas. Se discutirán los resultados, abordando primero una perspectiva metodológica y enseguida, una conceptual.

Desde la perspectiva metodológica, el análisis factorial indica la presencia de una estructura latente de cinco factores relacionados que presenta satisfactorios índices de ajuste, tanto a nivel exploratorio como confirmatorio, y es consistente con los planteamientos teóricos bajo los cuales se construyó el instrumento (Davidovich et al., 2005). Este hallazgo confirma empíricamente la multidimensionalidad y la naturaleza del constructo RS planteado previamente, tanto teórica 
Tabla 4. Ajuste de los modelos de invarianza según sexo y NSE

\begin{tabular}{|c|c|c|c|c|c|c|c|c|c|c|c|c|}
\hline & Modelo & $\chi^{2}(\mathrm{gl})$ & CFI & TLI & RMSEA & $90 \% \mathrm{IC}$ & $\begin{array}{l}\text { Comparación } \\
\text { de modelos }\end{array}$ & $\chi^{2}(\mathrm{gl})$ & $p$ & $\begin{array}{c}\Delta \\
\mathrm{CFI}\end{array}$ & $\begin{array}{c}\Delta \\
\text { TLI }\end{array}$ & $\begin{array}{c}\Delta \\
\text { RMSEA }\end{array}$ \\
\hline \multirow{4}{*}{ Sexo } & Configuración & $\begin{array}{l}2083.870 \\
(530)\end{array}$ & .962 & .957 & .055 & $\begin{array}{c}.053 \\
- \\
.058\end{array}$ & & & & & & \\
\hline & Métrico & $\begin{array}{c}2091.680 \\
\quad(550)\end{array}$ & .962 & .959 & .054 & $\begin{array}{c}.052 \\
- \\
.056\end{array}$ & $\begin{array}{l}\text { Métrico vs } \\
\text { Configuración }\end{array}$ & $\begin{array}{c}30.640 \\
(20)\end{array}$ & .060 & 0 & .002 & -.001 \\
\hline & Escalar & $\begin{array}{c}2203.553 \\
(620)\end{array}$ & .961 & .962 & .052 & $\begin{array}{c}.049 \\
- \\
.054\end{array}$ & $\begin{array}{l}\text { Escalar vs } \\
\text { Métrico }\end{array}$ & $\begin{array}{c}166.174 \\
(70)\end{array}$ & $<.001$ & -.001 & .003 & -.002 \\
\hline & Configuración & $\begin{array}{c}2027.196 \\
(530)\end{array}$ & .967 & .962 & .054 & $\begin{array}{c}.052 \\
- \\
.057\end{array}$ & & & & & & \\
\hline \multirow[t]{2}{*}{ NSE } & Métrico & $\begin{array}{c}2026.234 \\
\quad(550)\end{array}$ & .967 & .964 & .053 & $\begin{array}{c}.050 \\
- \\
.055\end{array}$ & $\begin{array}{l}\text { Métrico vs } \\
\text { Configuración }\end{array}$ & $\begin{array}{c}22.349 \\
(20)\end{array}$ & .321 & 0 & .002 & -.001 \\
\hline & Escalar & $\begin{array}{c}2096.666 \\
(620)\end{array}$ & .967 & .968 & .050 & $\begin{array}{c}.047 \\
- \\
.052\end{array}$ & $\begin{array}{l}\text { Escalar vs } \\
\text { Métrico }\end{array}$ & $\begin{array}{c}91.396 \\
(70)\end{array}$ & .044 & 0 & .004 & -.004 \\
\hline
\end{tabular}

$\mathrm{NSE}=$ Nivel Socioeconómico, gl = grados de libertad, 90\% IC = Intervalo de confianza al 90\%

Tabla 5. Estimaciones de fiabilidad para las dimensiones del ICOSORE-U

\begin{tabular}{|c|c|c|c|c|c|}
\hline \multirow{2}{*}{ Dimensión } & \multirow{2}{*}{ McDonald's $\omega$} & \multirow{2}{*}{ Greatest lower bound } & \multirow{2}{*}{ Cronbach's $\alpha$} & \multicolumn{2}{|c|}{$95 \%$ IC } \\
\hline & & & & Inferior & Superior \\
\hline Participación Religiosa & .919 & .930 & .918 & .906 & .918 \\
\hline Responsabilidad Académica & .663 & .675 & .658 & .620 & .672 \\
\hline Participación en Ayuda Social & .754 & .794 & .744 & .719 & .756 \\
\hline Convivencia Social & .654 & .728 & .651 & .606 & .656 \\
\hline Participación Ciudadana y Cultural & .629 & .694 & .626 & .599 & .652 \\
\hline
\end{tabular}

como empíricamente (Berman, 1997; Davidovich, 2005; Navarro, 2012). No obstante, difiere de las tres dimensiones del CACSR reformulado por Navarro et al. (2012). Estas diferencias pueden ser explicadas tanto por el número de ítems considerados en el ICOSORE-U, como por los nuevos análisis utilizados que consideran desarrollos estadísticos más recientes; en particular, el uso de una matriz de correlación policórica, recomendada en caso de trabajar con ítems ordinales (Holgado-Tello, ChacónMoscoso, Barbero-García, \& Vila-Abad, 2010), así como también el uso de métodos de estimación adecuados para esta matriz (Flora \& Curran, 2004; Flora et al., 2012).

En relación a las saturaciones factoriales, se observa que los ítems de cada una de las cinco escalas encontradas, presentan saturaciones directas y significativas con cada una de las dimensiones a las cuales tributan, con la excepción del ítem 1 que, en los análisis exploratorios, mide tanto el factor Convivencia Social como la Participación en Ayuda Social, condición que necesita ser estudiada con mayor profundidad para tomar una decisión final sobre su permanencia en virtud de mejorar la medida de RS.

El resto de los ítems muestran una clara tributación a una dimensión, indicando, por un lado, una relación lineal y, por otro, que las cinco dimensiones de la estructura latente son aspectos diferenciados de la manifestación conductual de la RS en este grupo evolutivo.

Respecto a la fiabilidad, los resultados encontrados pueden considerarse satisfactorios para casi todas las dimensiones del inventario, presentando elevada fiabilidad las dimensiones de Participación Religiosa y Participación en Ayuda Social, dado su contenido homogéneo; mientras que las demás dimensiones presentan niveles de fiabilidad más bajos, todas sobre .60. Si bien el valor de fiabilidad de estos factores es menor a los criterios de corte tradicionales (.70 o .80), Cho y Kim (2015), señalan que el valor de corte aceptable dependerá de la etapa de la investigación así como de los propósitos y consecuencias que el puntaje obtenido en el factor pueda tener en la vida del evaluado. En el presente 
estudio se trabaja con un instrumento nuevo, con escasa evidencia psicométrica previa, y con el cual no se tomarán decisiones directas que afecten la vida de las personas evaluadas, por tanto, estos niveles de fiabilidad pueden ser considerados adecuados. Ellos pueden ser atribuidos a que estas dimensiones incorporan aspectos diversos de la conducta evaluada, como en el caso, por ejemplo, de la Participación Ciudadana y Cultural que incluye dos ámbitos diferentes de participación.

Un avance metodológico en la evaluación de la fiabilidad consistió en incorporar no solo el coeficiente alfa de Cronbach, sino también otros estimadores más adecuados a las características de los datos en psicología, tales como el coeficiente omega y el GLB (Trizano-Hermosilla \& Alvarado, 2016), entregando consecuentemente estimaciones menos sesgadas de la fiabilidad de las dimensiones.

Otro avance metodológico, en cuanto a la profundización de las evidencias de validez del inventario, fue la evaluación del nivel de invarianza entre hombres y mujeres y entre los NSE medio-bajo y medio-alto para el modelo de cinco factores latentes. Los resultados permiten aceptar el máximo nivel de invarianza de umbrales (invarianza escalar), es decir, existe igualdad de estructura, igualdad de pesos factoriales e igualdad de umbrales entre ambos grupos. Sin embargo, lo anterior, se observaron diferencias de medias latentes, entre hombres y mujeres, para cuatro dimensiones y diferencias entre los NSE medio-bajo y medio-alto para tres dimensiones.

No obstante los avances en validez y las evidencias iniciales respecto de sexo y NSE, este estudio muestra varias limitaciones metodológicas. En primer lugar, si bien la muestra es numerosa y permite estimar con precisión los parámetros de los modelos factoriales, el muestreo no fue probabilístico, por lo tanto, se desconoce su grado de representatividad. Otra limitación, se refiere a la eliminación de cuatro ítems, justificada por su escasa relación teórica y empírica entre la definición de las dimensiones y el contenido de esos ítems. Considerando lo anterior, y ante la posibilidad de que la eliminación de esos ítems afecten las respuestas al instrumento, será necesario efectuar estudios que comprueben la estabilidad del instrumento sin los ítems que fueron eliminados.

Finalmente, se requiere desarrollar estudios que generen baremos de las puntuaciones mediante percentiles para cada una de las dimensiones, diferenciadas según sexo debido a las diferencias de medias latentes entre hombres y mujeres.

Desde la perspectiva conceptual, los resultados obtenidos en este estudio reafirman que las conductas estudiadas bajo el concepto de responsabilidad social corresponden, como planteaba Aristóteles, a la materialización de actos virtuosos que reflejan conductas pro-sociales, cooperativas, de participación y de autocontrol (Bravo, 2006; Navarro, 2012; Churchland \& Suhler, 2014; Carbonero et. al., 2017). Conductas, que, si bien son ejercidas individualmente, como, por ejemplo, "cuidé los espacios públicos como plazas, jardines, parques y monumentos" tienen un impacto en los demás y en la vida común, confirmando que la RS es un compromiso personal con los otros, pero también, un compromiso social con otros (Berman, 1997; Jonas, 2006). La identificación empírica de una estructura multidimensional apoya la concepción de que la RS contempla un ejercicio en diferentes dimensiones de la vida de los universitarios, como son los ámbitos: religioso, ciudadano, social, cultural y también académico (Navarro, 2012).

En cuanto a las diferencias encontradas en la frecuencia de comportamiento socialmente responsable en cuatro de las cinco dimensiones medidas, entre hombres y mujeres, son similares a los hallazgos encontrados en las mediciones con los instrumentos que sirvieron de referencia de este inventario (Navarro et al., 2010). Ellas pueden ser entendidas a partir, de un proceso de desarrollo femenino diferenciado del desarrollo masculino, que resulta en una construcción moral enfocada a la Ética de Cuidado propuesta por Chodorow (1978), Gilligan (1982) y Gilligan \& Attanucci (1988). Los autores postulan que las mujeres se definen a sí mismas en relación con el mundo, describiéndose por medio de acciones que las ponen en contacto con las demás. Para Chodorow (1978), el yo femenino es delimitado por la conexión con otros. Las mujeres elaboran nexos prestando y recibiendo ayuda, luego sus decisiones y acciones tienen presente la premisa 
de que nadie sea perjudicado; por el contrario, todos sean cuidados, incluidos y respetados (Gilligan, 1982; Gilligan \& Attanucci, 1988).

Por último, aunque no existen antecedentes previos de la relación entre NSE y conducta socialmente responsable, es posible encontrar otros estudios que revelan diferencias entre los jóvenes chilenos de distintos NSE en las dimensiones Participación Ciudadana y Cultural y Participación Religiosa que van en la misma dirección de los hallazgos presentados. Específicamente, en relación a la Participación Ciudadana y Cultural, sucesivos estudios del Instituto Nacional de la Juventud (INJUV, 2015, 2018), revelaron que quienes más participan de actividades políticas y sociales son los jóvenes de NSE alto. La misma encuesta en 2015, revela que los grupos de jóvenes que se sienten más identificados con alguna religión son de NSE bajo. Una posible explicación a estos resultados es que los jóvenes de NSE alto tienen más recursos personales, materiales y temporales para participar activamente en ámbitos ciudadanos y culturales, y por lo mismo, muestran menos identificación con la iglesia y la religión. Sin duda, esta es una relación que requiere más estudio $\mathrm{y}$ profundización.

En conclusión, este estudio no solo dio origen a un nuevo inventario con una estructura de cinco factores y satisfactorias propiedades psicométricas que refuerzan su utilidad y precisión mediante nuevas evidencias de fiabilidad y validez para ser usado en la medición de la RS en universitarios. También, este estudio y las modificaciones contenidas en el ICOSORE-U contribuyen a resolver la controversia existente sobre la estructura factorial del constructo RS y, por tanto, de la conducta socialmente responsable, estableciendo que se trata de una conducta compleja, que se manifiesta en múltiples dimensiones de la vida personal y social de los individuos. Por último, estas características aseguran la disposición de un instrumento de medida de la RS, que podrá ser utilizado en investigación y en la práctica educativa para el diagnóstico y seguimiento de los planes de formación de ciudadanos socialmente responsables en las universidades; además, su disponibilidad posibilita continuar su refinamiento psicométrico mediante estudios que consideren variables externas como criterios de validación externos, más allá de los adelantos preliminares del presente estudio.

\section{Referencias}

Asun, R. A., Rdz-Navarro, K., \& Alvarado, J. M. (2016). Developing multidimensional likert scales using item factor analysis: The case of four-point items. Sociological Methods \& Research, 45(1), 109-133. doi.org/1.1177/0049124114566716

Berman, S. (1990). The real ropes course: The development of social consciousness. ESR Journal, 1, 1-18.

Berman, S. (1997). Children's social consciousness and the development of social responsibility. New York State University Pressk.

Bravo, F. (2006). Teoría aristotélica de la responsabilidad. Estud.filos [online] 32, 109132.

Bronfenbrenner, U. (1979). The Ecology of Human Development. Cambridge, MA: Harvard University Press.

Carbonero, M. A., Martín-Antón, L. J., Otero, L., \& Monsalvo, E. (2017). Program to promote personal and social responsibility in the Secondary classroom. Front Psychol, 8, 809. doi:1.3389/fpsyg.2017.00809

Cho, E., \& Kim, S. (2015). Cronbach's coefficient alpha: Well known but poorly understood. Organizational Research Methods, 18(2), 207-23.

Chodorow, N. (1978). The reproduction of mothering. Berkeley: University of California Press.

Churchland, P. S., \& Suhler, C. L. (2014). Agency and control: The subcortical role in good decisions. En Walter Sinnott-Armstrong (Ed.). Moral Psychology: Free Will and Moral Responsibility (Vol.4). Cambridge, MA: The MIT Press.

Crocetti, E., Jahromi, P., \& Meeus, W. (2012). Identity and civic engagement in adolescence. Journal of Adolescence, 35, 521-532.

Davidovich, M. P., Espina, A., Navarro, G., \& Salazar, L. (2005). Construcción y estudio piloto de un cuestionario para evaluar 
comportamientos socialmente responsables en estudiantes universitarios. Revista de Psicología de la Universidad de Chile, 14(1), 125-139.

Eisenberg, N., \& Mussen, P. H. (1989). Cambridge studies in social and emotional development. The roots of prosocial behavior in children. New York, NY, US: Cambridge University Press.

Flora, D. B., \& Curran, P. J. (2004). An empirical evaluation of alternative methods of estimation for confirmatory factor analysis with ordinal data. Psychological Methods, 9(4), 466-491. doi.org/1.1037/1082-989X.9.4.466

Flora, D. B., LaBrish, C., \& Chalmers, R. P. (2012). Old and new ideas for data screening and assumption testing for exploratory and confirmatory factor analysis. Frontiers in Psychology, 3(MAR) 1-21. doi.org/1.3389/fpsyg.2012.00055

Gilligan, C. (1982). In a Different Voice. Cambridge, MA: Harvard University Press.

Gilligan, C., \& Attanucci, J. (1988). Two moral orientations: Gender differences and similarities. Merryl Palmer Quarterly, 34(3), 223-237.

Holgado-Tello, F. P., Chacón-Moscoso, S., Barbero-García, I., \& Vila-Abad, E. (2010). Polychoric versus pearson correlations in exploratory and confirmatory factor analysis of ordinal variables. Quality and Quantity, 44(1), 153-166. doi.org/1.1007/s11135-008-9190-y

Instituto Nacional de la Juventud. (2015). Octava Encuesta Nacional de la Juventud. Recuperado de http://www.injuv.gob.cl/storage/docs/Libro_O ctava_Encuesta_Nacional_de_Juventud.pdf

Instituto Nacional de la Juventud. (2018). Novena Encuesta Nacional de la Juventud. Recuperado de http://www.injuv.gob.cl/storage/docs/9\%C2\% B0_Encuesta_Nacional_de_Juventud_2018.p df

Jonas, H. (2006). O princípio responsabilidade: Ensaio de uma ética para a civilização tecnológica. Rio de Janeiro: Contraponto: Ed. PUC - Rio.

Kohlberg, L. (1984). Essays on Moral
Development (Vol. 2). San Francisco: Harper \& Row.

Kohlberg, L., \& Candee, D. (1984). The Psychology of Moral Development (Vol. 2). San Francisco: Harper \& Row.

Kueh, Y.C., Abdullah, N., Kuan, G., Morris, T., \& Naing, N.N. (2018) Testing measurement and factor structure invariance of the Physical Activity and Leisure Motivation Scale for Youth Across Gender. Front. Psychol. 9:1096. doi:1.3389/fpsyg.2018.01096.

Lalande, A. (1966). Vocabulario técnico y crítico de la filosofía. Buenos Aires: Editorial El Ateneo.

Ledesma, R. D., Ferrando, P. J., \& Tosi, J. D. (2019). Uso del Análisis Factorial Exploratorio en RIDEP. Recomendaciones para Autores y Revisores. Revista Iberoamericana de Diagnóstico y Evaluación - e Avaliação Psicológica,3(52), 173181. doi.org/1.21865/RIDEP52.3.13

Navarro, G. (2006). Comportamiento socialmente responsable. En Responsabilidad social universitaria, una manera de ser universidad, teoría y práctica de la experiencia chilena. Santiago, Chile: Proyecto Universidad Construye País.

Navarro, G. (2012). Moralidad y responsabilidad social: Bases para su desarrollo y educación. Concepción: Universidad de Concepción.

Navarro, G., Boero, P., Jiménez, G., Tapia, L., Hollander, R., Escobar, A., Baeza, M., \& Espina, A. (2010). Universitarios y responsabilidad social. Revista Calidad en la Educación, 33, 101-121.

Navarro, G., Pérez, C., \& Espina, A. (Octubre, 2012). Propiedades psicométricas del Inventario de Comportamientos Socialmente Responsables. Trabajo presentado en la VII Encuentro Nacional de Educación para la Responsabilidad Social, Universidad de Concepción, Concepción, Chile.

Organización de las Naciones Unidas para la Educación, la Ciencia y la Cultura (1998). Declaración mundial sobre la educación superior en el siglo XXI: Visión y acción. Recuperado de http://www.unesco.org/education/educprog/w che/declaration_spa.htm

Oros, L. B. (2017). Valores Normativos de la 
Escala Multidimensional de Autoeficacia Infantil para Población Argentina. Revista Iberoamericana de Diagnóstico y Evaluación - e Avaliação Psicológica, 2(44), 172-181. doi.org/1.21865/RIDEP44.2.14

Piaget, J. (1932/1994). O juízo moral na criança. Sao Paulo: Summus.

Putnick, D. L., \& Bornstein, M. H. (2016). Measurement invariance conventions and reporting: The State of the Art and Future Directions for Psychological Research. Developmental Review: DR, 41, 719. doi.org/1.1016/j.dr.2016.06.004

Reise, S. P. (2012). The rediscovery of bifactor measurement models. Multivariate Behavioral Research, 47(5), 667-696. https://doi.org/1.1080/00273171.2012.715555

Revelle, W., \& Zinbarg, R. (2009). Coefficients alpha, beta, omega, and the glb: Comments on Sijtsma. Psychometrika, 74(1), 145-154.

Schreiber, J., Nora, A., Stage, F., Barlow, E., \& King, J. (2006). Reporting structural equation modeling and confirmatory factor analysis results: A review. The Journal of Educational Research, 99(6), 323-337. Recuperado de doi/abs/1.3200/JOER.99.6.323-338

Sijtsma, K. (2009). On the use, the misuse, and the very limited usefulness of Cronbach's alpha. Psychometrika, 74(1), 107-12.

Trizano-Hermosilla, I., \& Alvarado, J. M. (2016). Best alternatives to cronbach's alpha reliability in realistic conditions: Congeneric and asymmetrical measurements. Frontiers in Psychology, 7(May), 1-8. doi.org/1.3389/fpsyg.2016.00769

Tuning América Latina (2007) Reflexiones y perspectivas de la educación superior en América Latina. Recuperado de http://tuningacademy.org/wpcontent/uploads/2014/02/TuningLAIII_FinalReport_SP.pdf

Yang, Y., \& Green, S. B. (2011). Coefficient alpha: A reliability coefficient for the $21 \mathrm{st}$ century? Journal of Psychoeducational Assessment, 29(4), 377-392. doi.org/1.1177/0734282911406668

Zinbarg, R. E., Revelle, W., Yovel, I., \& Li, W. (2005). Cronbach's $\alpha$, Revelle's $\beta$, and Mcdonald's $\omega \mathrm{H}$ : Their relations with each other and two alternative conceptualizations of reliability. Psychometrika, 70(1), 123-133. doi.org/1.1007/s11336-003-0974-7

Zinbarg, R. E., Yovel, I., Revelle, W., \& McDonald, R. (2006). Estimating generalizability to a latent variable common to all of a scale's indicators: A comparison of estimators for $\omega \mathrm{h}$. Applied Psychological Measurement, 30(2), 121-144. doi.org/1.1177/0146621605278814 


\section{Anexo A}

Dimensiones e ítems Inventario de Conductas Socialmente Responsables

\begin{tabular}{|c|c|c|}
\hline Participación en Ayuda Social & Convivencia Social & $\begin{array}{c}\text { Participación ciudadana y } \\
\text { cultural }\end{array}$ \\
\hline $\begin{array}{l}\text { Conductas solidarias que directa } \\
\text { o indirectamente atienden y } \\
\text { satisfacen las carencias y } \\
\text { necesidades de los otros. }\end{array}$ & $\begin{array}{l}\text { Conductas que incluyen el } \\
\text { respeto y cuidado por los demás } \\
\text { acorde con las normas y } \\
\text { necesidades individuales y } \\
\text { grupales de la vida en comunidad. }\end{array}$ & $\begin{array}{l}\text { Conductas de participación } \\
\text { cultural e institucional que } \\
\text { tributan a la vida ciudadana } \\
\text { activa. }\end{array}$ \\
\hline $\begin{array}{l}\text { 1. Me ocupé del medio ambiente } \\
\text { (recolección de basura, limpieza } \\
\text { de lugares públicos) o participé } \\
\text { en una organización que se ocupa } \\
\text { del medio ambiente. } \\
\text { 3. Participé en actividades de } \\
\text { voluntariado como: construir } \\
\text { mediaguas, visitar hogar de niños } \\
\text { o de ancianos, realizar trabajos } \\
\text { voluntarios de verano o invierno, } \\
\text { entre otros. } \\
\text { 10. Organicé o ayudé a organizar } \\
\text { campañas de ayuda solidaria, } \\
\text { tales como: recolectar alimentos, } \\
\text { vestimenta, útiles escolares, entre } \\
\text { otros. } \\
\text { 21. Participé o ayudé en una } \\
\text { organización de caridad como: } \\
\text { Hogar de Cristo, Ejército de } \\
\text { Salvación, Fundación Las Rosas, } \\
\text { Regazo, entre otras. } \\
\text { 26. Entregué recursos materiales } \\
\text { a campañas de ayuda social, } \\
\text { como: vestimentas, alimentos, } \\
\text { materiales de construcción, entre } \\
\text { otros. }\end{array}$ & $\begin{array}{l}\text { 7. Boté la basura en los basureros } \\
\text { (de la calle, de los parques o de } \\
\text { lugares públicos). } \\
\text { 8. Cuidé los espacios públicos } \\
\text { como plazas, jardines, parques, } \\
\text { monumentos. } \\
\text { 14. Utilicé racionalmente los } \\
\text { recursos como el agua, la luz, el } \\
\text { gas, cuidar los espacios naturales, } \\
\text { entre otros. } \\
\text { 19. Respeté los espacios } \\
\text { reservados para minusválidos, } \\
\text { embarazadas y/o ancianos. } \\
\text { 23. Respeté las normas sociales } \\
\text { tales como: respetar la fila, } \\
\text { turnos, ceder el asiento a personas } \\
\text { mayores. } \\
\text { 25. Cuidé instalaciones públicas o } \\
\text { universitarias (infraestructura, } \\
\text { mobiliario, libros). } \\
\mathbf{2 9} \text {. Evité usar productos que } \\
\text { contaminan el medio ambiente } \\
\text { como aerosoles, envases no } \\
\text { reciclables, tubos de escape en } \\
\text { mal estado, entre otros. }\end{array}$ & $\begin{array}{l}\text { 6. Me informé sobre el acontecer } \\
\text { nacional e internacional (ver } \\
\text { noticias, leer el diario, revisar } \\
\text { medios electrónicos). } \\
\text { 17. Participé en algún } \\
\text { movimiento estudiantil u } \\
\text { organización política (partido } \\
\text { político, federación de } \\
\text { estudiantes, centro de alumnos, } \\
\text { asambleas, entre otros). } \\
\mathbf{2 4} \text {. Voté en elecciones de alguna } \\
\text { institución u organización a la } \\
\text { que pertenezco, como, por } \\
\text { ejemplo, votaciones de carrera, de } \\
\text { organizaciones ciudadanas, } \\
\text { municipales parlamentarias o } \\
\text { presidenciales. } \\
\text { 27. Expresé mi opinión respecto } \\
\text { de distintos temas, en diferentes } \\
\text { contextos y con diferentes } \\
\text { personas. } \\
\text { 28. Participé en actividades } \\
\text { culturales (conciertos, obras de } \\
\text { teatro, exposiciones de pintura, } \\
\text { escultura, entre otras). }\end{array}$ \\
\hline
\end{tabular}

Dimensiones e ítems Inventario de Conductas Socialmente Responsables

\begin{tabular}{|c|c|}
\hline Participación Religiosa & Responsabilidad Académica \\
\hline $\begin{array}{l}\text { Conductas vinculadas al ejercicio y transmisión de } \\
\text { los propios valores religiosos. }\end{array}$ & $\begin{array}{l}\text { Conductas compatibles con las exigencias y } \\
\text { oportunidades que tiene la vida universitaria. }\end{array}$ \\
\hline $\begin{array}{l}\text { 4. Participé en algún grupo religioso (cualquier } \\
\text { religión) o algún grupo ligado a la iglesia (cualquier } \\
\text { iglesia). } \\
\text { 11. Participé en las actividades propias de la religión } \\
\text { que profeso. } \\
\text { 16. Invité a otras personas a participar en actividades } \\
\text { de mi grupo religioso. } \\
\text { 22. Transmití mis valores religiosos a otras personas. }\end{array}$ & $\begin{array}{l}\text { 2. Asistí a clases o a mi trabajo. } \\
\text { 9. Cumplí responsablemente con trabajos, tareas } \\
\text { y evaluaciones. } \\
\text { 15. Estudié las asignaturas de mi carrera o me } \\
\text { perfeccioné en mi trabajo. } \\
\text { 20. Llegué puntualmente a mis actividades } \\
\text { académicas y/o laborales (reuniones de trabajo, } \\
\text { clases, prácticas profesionales, certámenes, entre } \\
\text { otros). }\end{array}$ \\
\hline
\end{tabular}

